\title{
Spectral Tuning of the Long-Period Fiber Gratings by Using Low Birefringence Liquid Crystals
}

\author{
A. Czapla ${ }^{a, b, *}$, W.J. Bock ${ }^{a}$, T.R. Woliński ${ }^{b}$, R. DAzBrowski ${ }^{c}$ \\ AND E. NOWINOWSKI-KRUSZELNICKI ${ }^{c}$ \\ ${ }^{a}$ Centre de Recherche en Photonique, Université du Québec en Outaouais \\ 101 rue Saint-Jean-Bosco, Gatineau, Québec J8X 3X7, Canada \\ ${ }^{b}$ Faculty of Physics, Warsaw University of Technology, Koszykowa 75, 00-662 Warszawa, Poland \\ ${ }^{c}$ Military University of Technology, Gen. S. Kaliskiego 2, 00-908 Warszawa, Poland
}

\begin{abstract}
The work presented in this paper is focused on investigation of the spectral properties of the long-period fiber gratings combined with the liquid crystals, named liquid crystal long-period fiber gratings. The experiments carried out showed that the proposed designs of the liquid crystal long-period fiber gratings can offer very interesting spectral properties and can introduce a new level of sensitivity. In particular, a high-efficiency thermal tuning of the long-period fiber gratings coated with low-birefringence liquid crystal layers could be achieved and gave rise to a fast and wide switching ability of the attenuation bands within their transmission spectrum.
\end{abstract}

PACS: 42.81.Cn, 42.79.Dj, 42.81.Pa, 42.79.Kr

\section{Introduction}

Optical fibers functionalized with periodic micro-scale structures appear as key elements in sensing components thanks to new possibilities for light guiding and control. One of the most matured classes of this kind of devices is a long-period fiber grating (LPFG) $[1,2]$. These grating devices act as narrow band-stop filters. Within a relative short time it became obvious that the LPFGs could be successfully incorporated into a fiber optic sensing system $[3,4]$. This is due to the fact that they provide a mechanism for producing a wavelength-dependent attenuation in the transmission, which is sensitive to the external effects. Furthermore, tuning properties of the LPFGs have been exploited by combining them with the liquid crystals (LCs). LCs are self-organized anisotropic materials that exhibit high electro-optic and thermo-optic effects associated with their birefringence, with their dielectric anisotropy and with thermal dependence of their refractive indices [5-7]. In this context the use of the LCs has gained a particular attention resulting in a class of new sensing components, later named liquid crystal long-period fiber gratings (LC-LPFGs) [8-11].

In this paper we present an experimental study of the LPFG coated with a thin LC layer. As an "active" LC material we used a prototype low-birefringence (LB) nematic 1550 LC mixture. A unique property of this LC material is that it has an ordinary refractive index $\left(n_{\mathrm{o}}\right)$ which can be higher than, equal to, or lower than the

\footnotetext{
* corresponding author; e-mail: czapla@if.pw.edu.pl
}

refractive index of silica in a certain range of temperatures. This feature of the LC layer external to the LPFG is explored in order to dynamically tune the propagation properties of the cladding modes.

\section{Principle of operation}

An LPFG is a grating with a pitch of several hundred micrometers formed in an optical fiber. In the LPFGs a periodic axial perturbation in the refractive index of the core generates a co-directional mode coupling between the propagating core mode and the forward propagating cladding mode. Since the cladding modes suffer from high attenuation, the transmission spectrum of the LPFG consists of a series of attenuation bands. The allowed resonant wavelength $\left(\lambda_{\text {res }}\right)$ of the mode coupling is given by the following condition:

$$
\lambda_{\text {res }}=\left(n_{\mathrm{co}}^{\mathrm{eff}}-n_{\mathrm{cl}, m}^{\mathrm{eff}}\right) \Lambda,
$$

where $n_{\mathrm{o}}^{\mathrm{eff}}, n_{\mathrm{cl}, m}^{\mathrm{eff}}$ stand for the effective refractive index of the core mode and the effective refractive index of the $m$-th cladding mode, and $\Lambda$ is the period of the grating. The value of the $\lambda_{\text {res }}$ depends on the value of the surrounding refractive index (SRI) [12]. Thus, as far as the LPFG surrounded by an LC is concerned, one can expect that tuning of the $\mathrm{LC}$ refractive index $\left(n_{\mathrm{LC}}\right)$ will induce a modification of the spectral response of the LPFG. In addition, the propagation mechanism of the cladding modes is affected by the difference between the refractive indices of the fiber cladding $\left(n_{\mathrm{cl}}\right)$ and the LC surrounding medium 


$$
\Delta n_{\mathrm{cl}, \mathrm{LC}}=n_{\mathrm{cl}}-n_{\mathrm{LC}} .
$$

Therefore, in order to study the spectral properties of the LC-LPFGs, they should be analyzed according to the difference given by Eq. (2). When $\Delta n_{\mathrm{cl}, \mathrm{LC}}>0$ the cladding modes experience a TIR mechanism at the interface between the cladding and the surrounding medium. When $\Delta n_{\mathrm{cl}, \mathrm{LC}}<0$ and an infinitely thick LC layer is assumed, the fiber cladding modes become leaky [13]. The existence of couplings at discrete wavelengths in this regime corresponds with the magnitude of the Fresnel reflection coefficients which dictate the proportion of light energy that is reflected. In this case, the tuning range of the LPFG with LC is limited mainly to the controlling of the intensity of attenuation bands induced by the variation of the LC refractive index. However, when $\Delta n_{\mathrm{cl}, \mathrm{LC}}<0$ and the LC layer is thick enough, due to the refractive-reflective regime at the interface between the cladding and the thin LC layer, the cladding modes in the LPFG can be bound within the structure comprising the core, the cladding, and the LC layer [14, 15].

\section{Materials and experimental setups}

The LPFGs for our experiments were fabricated by UV irradiation in a boron co-doped photosensitive fiber (PS1250/1500, manufactured by Fibercore). The period of the tested LPFGs was $227 \mu \mathrm{m}$ and their length was $4 \mathrm{~cm}$. To form an LC layer on the LPFG, first, the LPFG was rubbed several times along the fiber axis using cotton swabs so that the LC molecules will tend to align along the fiber axis. In reality, there exists a certain non-zero tilt angle of the LC molecules with respect to the fiber axis, which depends on the geometry of the LC layer as well as on the fiber itself. However, the overall effect can be negligible so that the effective refractive index of the LC layer can be approximated by the ordinary refractive index, $n_{\mathrm{o}}$. Then, the LPFGs were coated by a thin layer of LC. A surface tension of the LC makes it easy to coat uniformly the LPFGs making them to behave as if they had a skin. The presence of the LC layer on the surface of the LPFG was observed when the sample was placed between the parallel polarizers. Since the birefringence of tested LC mixture is known, the thickness of the LC layer was estimated to be of the order of $1 \mu \mathrm{m}$ - according to the Michel-Levy interference chart [16].

The experimental setup for conducting the temperature-induced measurements of the LPFGs with LC layers is presented in Fig. 1a. The section of the LPFG with LC layer was placed into a Pelletier module. Temperature regulation was within the $10-120^{\circ} \mathrm{C}$ range with the Testo 735 precise thermometer $\left(0.05^{\circ} \mathrm{C}\right.$ resolution and $0.1^{\circ} \mathrm{C}$ accuracy). Each measurement was performed after stabilization of the temperature by the Pelletier module. The transmission spectra of the samples were investigated with input light launched from a halogen lamp and the output signal analyzed by an optical spectrum analyzer with a maximum resolution of $0.05 \mathrm{~nm}$. As a material surrounding the LPFGs, a prototype LB LC mixture, named 1550, was chosen.

The temperature dependence of its refractive indices is presented in Fig. 1b. A unique feature of these LC mixtures is that they have $n_{\mathrm{o}}$, which can be thermally tuned within a wide range of values.
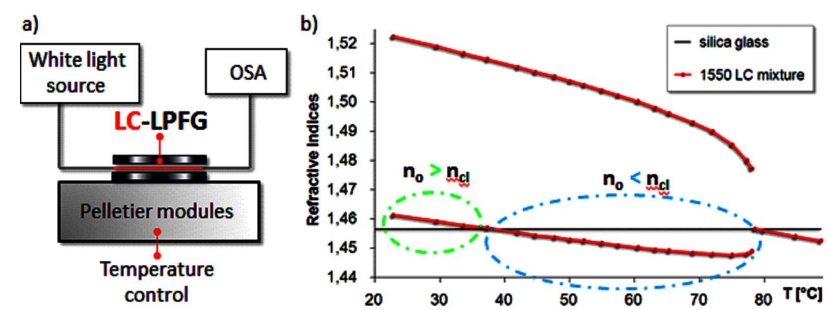

Fig. 1. (a) Experimental setup for temperature-induced measurements of the LC-LPFGs; (b) the temperature dependence of $1550 \mathrm{LC}$ mixture refractive indices.

\section{Experimental results and their analysis}

In Fig. 2 the transmission spectra of the UV-induced LPFG with a thin layer of 1550 LC mixture are presented for different temperatures. As can be seen, a strong modification of the transmission spectrum of this LC-LPFG occurs between the temperatures from $36^{\circ} \mathrm{C}$ to $49^{\circ} \mathrm{C}$ : one band at $1229.5 \mathrm{~nm}$, designated as band 1 , is split into two other bands, designated as band_1'a and band_1'b. In addition, one more band starts appearing, designated as band_2', and at $63^{\circ} \mathrm{C}$ it reaches the $\lambda_{\text {res }}$ of $1426 \mathrm{~nm}$. In order to understand the results presented in Fig. 2, two effects should be considered while the LC-LPFG was heated. The first one is related to the sensitivity of the grating to the LC refractive index changes induced by temperature. The second one is related to the host fiber properties. The grating studied here was fabricated within the Be/Ge co-doped fiber. Such an LPFG exhibits the spectral shifts towards the lower $\lambda_{\text {res }}$ with an increasing temperature [17]. Which of these two effects dominates will depend primarily on the magnitude of the inherent thermally sensitive LPFG host and on the relationship between the $n_{\mathrm{cl}}$ and the $n_{\mathrm{o}}$.

Additionally, depending on the difference between the cladding and LC layer refractive indices $\Delta n_{\mathrm{cl}, \mathrm{LC}}$ (given by Eq. (2)) the cladding modes propagate in a different manner. Therefore, the thermally induced changes in the transmission spectrum of the LPFG with 1550 LC layer should be analyzed in two separate temperature ranges.

\subsection{Case 1: The temperature range of operation is below $T_{\mathrm{NO}}$}

When the operating temperature was below $T_{\mathrm{NO}}$ $\left(T_{\mathrm{NO}}=43{ }^{\circ} \mathrm{C}\right.$ indicates the point in the thermal characteristic for $1550 \mathrm{LC}$ mixture where $n_{\mathrm{o}}$ is equal to the $n_{\mathrm{cl}}$ ), the difference between the cladding and LC layer refractive indices $\Delta n_{\mathrm{cl}, \mathrm{LC}}$ is negative. Due to the fact that 


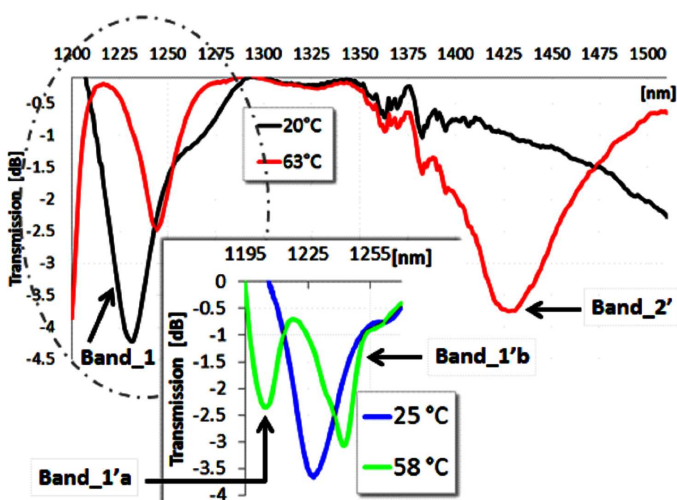

Fig. 2. Shaping the transmission spectrum of the LPFG_PS with 1550 LC mixture by variable temperature.

the thickness of the LC layer is approximately $1 \mu \mathrm{m}$, the cladding modes in an LC-LPFG are bound within the structure comprising the core, the cladding and the $\mathrm{LC}$ layer. In this case the thermally induced changes in the $n_{\mathrm{o}}$ can lead to a red shift of the attenuation bands in the transmission spectrum of the LC-LPFG (Fig. 3). From the other side, since the investigated grating is based on the $\mathrm{Be} / \mathrm{Ge}$ co-doped fiber, the LPFG exhibits blue shifts of the attenuation bands with an increasing temperature. As a result, the thermal tuning ability of the resonant wavelength $\lambda_{\text {res }}$ could be limited due to the present of the LC layer. In addition, the strength of a coupling mode is related to the magnitude of the $\Delta n_{\mathrm{cl}, \mathrm{LC}}$ (it decreases with temperature). Consequently, while the LC-LPFGs were heated, the depth of their attenuation band decreased.

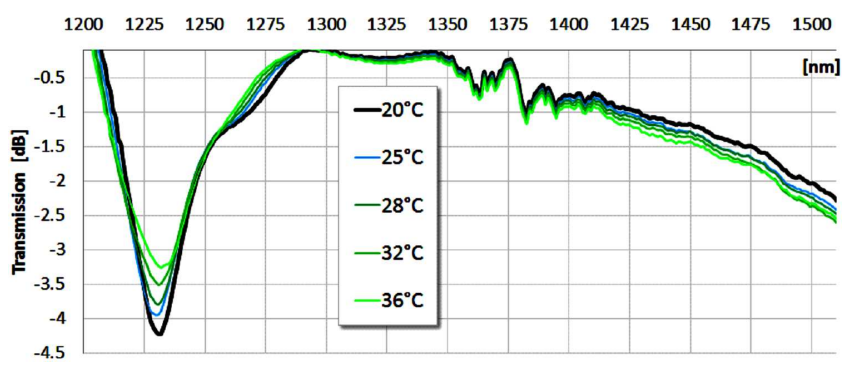

Fig. 3. Transmission spectra of the LPFGs with a 1550 LC layer versus temperature: the temperature range of operation is below $T_{\mathrm{NO}}$.

\subsection{Case 2: The temperature range of operation is above the $T_{\mathrm{NO}}$}

When the sample was heated above $T_{\mathrm{NO}}, \Delta n_{\mathrm{cl}, \mathrm{LC}}$ changed its sign to positive and the cladding modes started to experience a total internal reflection (TIR). As a result, three new bands appeared in the LC-LPFG transmission spectrum (Fig. 4). Further, these three adjacent bands gradually grow to be clearly observed in the transmission spectrum while temperature is being increased. This behavior can be explained by the fact that at this temperature range of operation the value of the $\Delta n_{\mathrm{cl}, \mathrm{LC}}$ increases with temperature and the strength of coupling modes becomes greater.

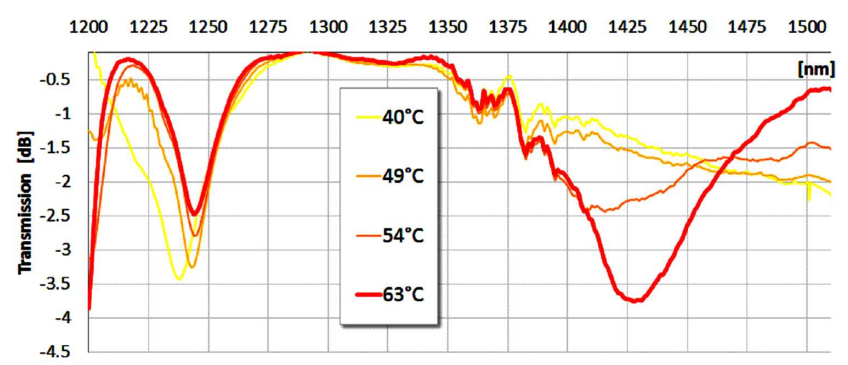

Fig. 4. Transmission spectra of the LPFG with a 1550 LC layer versus temperature: the temperature range of operation is above $T_{\mathrm{NO}}$.

Taking into consideration the analysis presented above, by operating at a temperature range close to $T_{\mathrm{NO}}$ of the LC, "switching" of the sign of $\Delta n_{\mathrm{cl}, \mathrm{LC}}$ (from negative to positive) for such LC-LPFGs can be achieved. This switching is possible due to the unique properties of the 1550 LC mixture whose $n_{\mathrm{o}}$ can be higher, equal, or lower than the $n_{\mathrm{cl}}$ in a certain range of temperatures. Consequently, tuning of the propagation properties of the cladding modes at the temperature where the $n_{\mathrm{o}}$ is equal with $n_{\mathrm{cl}}$ has been achieved. At this point a significant change of the shape of the LC-LPFG transmission spectrum would occur.

\section{Conclusions}

The experiments carried out showed that tuning properties of the LPFGs can be enhanced by functionalizing them with the LCs. We have demonstrated a grating combined with a prototype LB 1550 LC mixture. Due to special properties of the LC mixture used in this study, thermally-induced switching of the propagation properties of the cladding modes could be obtained: from the propagation in the thin LC layer to the propagation based on the TIR mechanism. Consequently, a strong modification of the transmission spectrum of the LC-LPFGs in a significant range of temperatures could be recorded.

The proposed and innovative design of the LC-LPFGs has simultaneously advanced research in the fields of both LPFGs and LCs by demonstrating that such optical components can be more useful than previously thought. As a result, new highly-tunable all in-fiber devices based on the LC-LPFGs can be obtained for various sensing applications.

\section{Acknowledgments}

This work was supported by the Natural Science and Engineering Research Council of Canada and by the Canada Research Chairs Program. The authors are also 
grateful to the Foundation for Polish Sciences (FNP) Professor's Subsidy "MISTRZ", to Warsaw University of Technology, and to the Fonds Québécois de la Recherche sur la Nature et les Technologies (FQRNT).

\section{References}

[1] T. Erdogan, J. Lightwave Technol. 15, 1277 (1997).

[2] A.N. Vengsarkar, P.J. Lemaire, J.P. Judkins, V. Bhatia, T. Erdogan, J.E. Sipe, J. Lightwave Technol. 14, 58 (1996).

[3] X. Shu, L. Zhang, I. Bennion, J. Lightwave Technol. 20, 255 (2002).

[4] V. Bhatia, Opt. Express 4, 457 (1999).

[5] I.C. Khoo, S.T. Wu, Optics and Nonlinear Optics of Liquid Crystals, World Sci., Singapore 1997.

[6] M.M. Tefelska, M.S. Chychłowski, T.R. Woliński, R. Dąbrowski, W. Rejmer, J. Wójcik, Acta Phys. Pol. A 118, 1259 (2010).

[7] M.S. Chychłowski, S. Ertman, M.M. Tefelska, T.R. Woliński, E. Nowinowski-Kruszelnicki, O. Yaroshchuk, Acta Phys. Pol. A 118, 1100 (2010).

[8] Hak-Rin Kim, Y. Kim, Y. Jeong, S. Baek, Y. Wook Lee, B. Lee, S.-D. Lee, Mol. Cryst. Liq. Cryst. 413, 399 (2004).
[9] O. Duhem, J.F. Henniont, M. Warenghem, M. Douay, L. Rivoallan, in: Proc. 6th IEE Conf. on Telecommunications, Vol. 451, Edinburgh 1998, p. 195.

[10] A. Czapla, T.R. Woliński, R. Dąbrowski, E. Nowinowski-Kruszelnicki, W.J. Bock, Photon. Lett. Poland 2, 116 (2010).

[11] A. Czapla, W.J. Bock, P. Mikulic, T.R. Wolinski, Bull. Pol. Acad. Sci. Techn. Sci. 58, 503 (2010).

[12] H.J. Patrick, A.D. Kersey, F. Bucholtz, J. Lightwave Technol. 16, 1606 (1998).

[13] R. Hou, Z. Ghassemlooy, A. Hassan, C. Lu, K.P. Dowker, Meas. Sci. Technol. 12, 1709 (2001).

[14] Z. Wang, J.R. Heflin, R.H. Stolen, S. Ramachandran, Opt. Express 13, 2808 (2005).

[15] I. Del Villar, I.R. Matías, F.J. Arregui, Opt. Express 13, 56 (2004).

[16] J.G. Delly, "The Michel-Lévy interference color chart. The microscopist's magical color key", on-line journal Modern Microscopy (2003).

[17] M. Śmietana, W.J. Bock, P. Mikulic, Meas. Sci. Technol. 21, 1 (2010). 